\title{
Bed shear stress estimation for gravity currents performed in laboratory
}

\author{
Jessica Zordan, Anton J. Schleiss \& Mário J. Franca \\ Laboratoire de Constructions Hydrauliques, École Polytechnique Fédérale de Lausanne, Switzerland
}

\begin{abstract}
Gravity currents are caused by density differences between two fluids which may be due to temperature, dissolved substances or the presence of particles in suspension. In this study saline currents, in which the higher density is produced by dissolved salt, are reproduced in laboratory with the aim to characterize the bed shear stress. Saline currents can in fact be responsible for high erosion rates and the bed shear stress is a quantification of this erosive capacity. The dynamics of buoyancy driven flows are complex and the effect of the initial density gravity current on the bed shear stress is not explored yet. The results herein showed confirm the importance of detailed velocity profile measurements for the determination of the friction velocity which is a key parameter for the currents propagation and for characterizing the momentum and mass exchanges between the current and the bed. The spatial evolution of the bed shear stress caused by the passage of a gravity current is here estimated using the logarithmic velocity profile method for, as a first attempt, a value of the von Kármán constant of $k=0.405$. The use of this constant is then verified and discussed.
\end{abstract}

\section{INTRODUCTION}

Gravity currents are buoyancy driven flows that occur, naturally or as a consequence of human activities, due to temperature differences, dissolved substances or to the presence of particles in suspension (Simpson 1997). Examples of gravity currents in water are oceanic fronts, resulting from differences in temperature and salinity, and turbidity currents caused by high concentration of suspended particles. Gravity currents have been widely studied through both numerical (Ooi et al. 2009, Adduce et al. 2012, Ottolenghi et al. 2014, Lombardi et al. 2015) and experimental (Huppert \& Simpson 1980, Altinakar et al. 1990, Garcia \& Parker 1993, Shin et al. 2004, Britter \& Linden 2006, Nogueira et al. 2013, Nogueira et al. 2014) simulations. In spite a wide presence in literature, just few contributions are devoted to the determination of bed shear stress of gravity currents.

The majority of turbulence production comes from the shear at the lower boundaries where the gradient of the mean streamwise velocity is higher (Gray et al. 2006). When the passage of the gravity current is made over a mobile bed, entrainment and detrainment (if the current is charged with sediments) may take place dependent on the bed shear stress, particle grain size and density (Garcia \& Parker 1993). The level of exchange of sediments with the bed determines the lifetime of the current according to the autosuspention criteria (Parker et al. 1986). The magnitude of the shear stress at the lower boundary layer determines the sediment transport capacity of saline currents and whether erosion or deposition processes dominate the regime at the bottom boundary (Cossu \& Wells 2012).

Here, four gravity currents of different initial density are performed in laboratory, in a set-up specifically design to perform full-depth lockexchange experiments. The standard configuration that was used by such authors as Huppert \& Simpson (1980), Rottman and Simpson (1983), Adduce et al. (2012), Nogueira et al. (2014), among others is here revised: the lock gate separates the two fluids of different densities in a horizontal channel where the initial volume of heavier fluid is comparable to the volume of the ambient water in the second part of the channel (Shin et al. 2004). This configuration allows the formation of an extended slumping phase in which the front velocity is almost constant (Tokyay et al. 2011). In these conditions, a quasi-steady regime is formed, similar to the steady state observed for constant feed gravity currents. Once the flow becomes steady, one can use methods developed to analyze constant-density channel flow for the study of the turbulence structure (Tokyay et al. 2012).

Bed shear stress $\tau_{b}$ is not measured directly but it is inferred from the friction velocity $u_{*}$. Due to the quadratic relationship between $\tau_{b}$ and $\mathrm{u}_{*}$, high-quality estimates of $u_{*}$ are required in order to obtain reliable bed shear stress estimations (Bagherimiyab \& Lemmin 2013). The near boundary region is the most difficult layer from which to obtain reliable velocities measurements since it is where the higher velocity gradients are located 
(Yang et al. 2012). A number of methods are available for estimating friction velocity: logarithmic mean velocity profile, Turbulent Kinetic Energy (TKE) profile, wall similarity and spectral methods among others. In the present study friction velocity was estimated using the instantaneous velocity measurements that an Acoustic Doppler Velocity Profiler records along a profile. The ADVP is a high-resolution non-intrusive instrument that can record quasi-instantaneous velocities measurements along a profile. The evolution of the bed shear stress caused by the passage of a gravity current is here calculated by using the logarithmic velocity profile method.

The present paper is structured as such: first of all the experimental set-up, the instrumentations and the procedure are described. Then a method for the estimation of the bed shear stress is explained. The results (longitudinal velocity and bed shear stress) are shown and discussed. Finally, the main findings are summarized in the concluding section.

\section{METHODS}

\subsection{Experimental set-up}

The laboratory set-up was specifically designed to reproduce saline bottom density currents. The flume is $7.5 \mathrm{~m}$ long and $0.275 \mathrm{~m}$ wide and it is divided into two sections of comparable volumes by a vertically sliding gate (Fig. 1). The bottom is horizontal and smooth. An upstream reach serves as head tank for the dense mixture; a downstream reach is where the current propagates and where the main measurements are made. The so called lock exchange technique is used: when the gate is removed, differences in the hydrostatic pressure cause the denser fluid to flow in one direction near the bottom boundary of the tank, while the lighter fluid flows in the opposite direction at the top (Shin et al. 2004). Downstream, the current is let to dissipate flowing down into a final large tank.

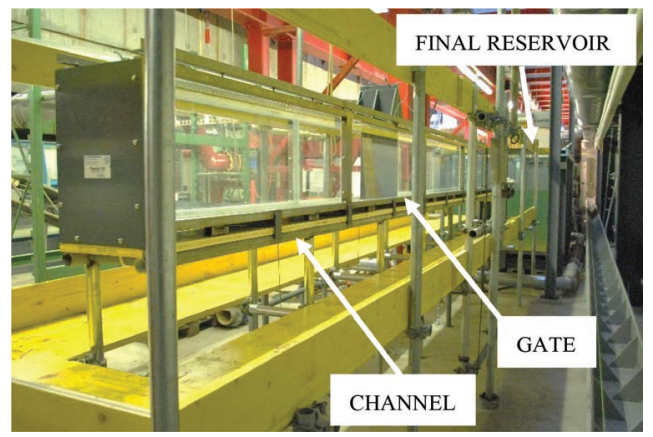

Figure 1. Experimental set-up at LCH (EPF Lausanne).

\subsection{Measurements and instrumentation}

The 3D Acoustic Doppler Velocity Profiler (ADVP) (Lemmin \& Rolland 1997, Franca \& Lemmin 2006) is a non-intrusive sonar instrument that measures the instantaneous velocity profiles using the Doppler effect without the need of calibration. It is placed in the second half of the channel on a sliding structure (Fig. 2) and takes 3D instantaneous velocity measurements during the passage of the density current over a vertical, including the upper counter flow. For studies of turbulent flow, a high sampling frequency is desirable. The minimum number of pulse-pairs was here fixed at 32, in reason of our working conditions, which corresponds to a frequency of acquisition of $31.25 \mathrm{~Hz}$ (Lemmin \& Rolland 1997). The instrument consists of a central emitter surrounded by four receivers. The geometric configuration is the result of an optimization of the instrument that allows noise reduction by creating redundancy information for the velocity components (Blanckaert \& Lemmin 2006). This, together with the despiking procedure proposed by Goring \& Nikora (2002), leads to a considerable reduction in the noise level of the data set. Instantaneous longitudinal velocity profiles characterizing the body of the current are here analyzed. An upscaling technique of instantaneous velocity has been chosen for handling fine

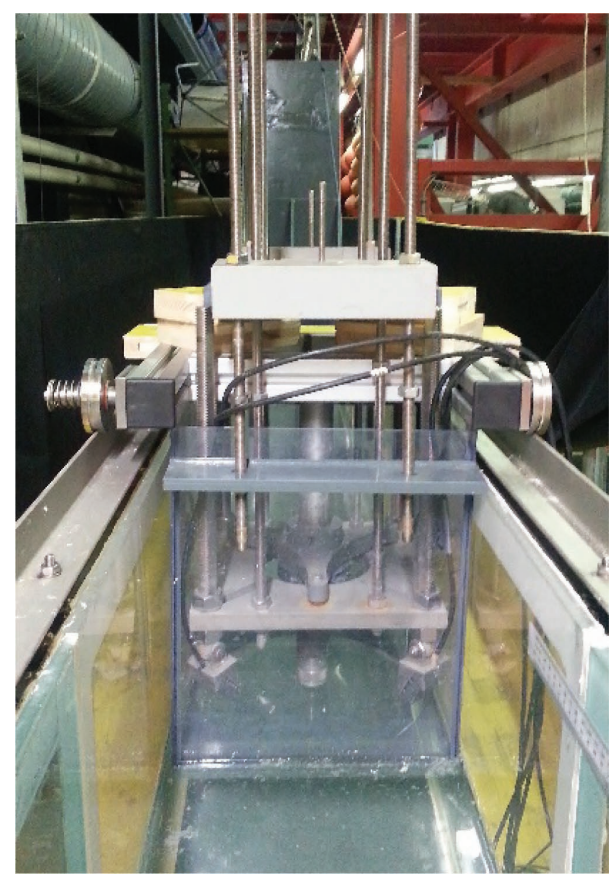

Figure 2. The ADVP (Acoustic Doppler Velocity Profiler) installed in the channel. 
scale heterogeneities in order to apply the log-law method over a long enough quasi-steady period (see Section 2.5).

Before each test, the initial density of ambient fluid and of the heavier fluid were measured with a densimeter, together with the temperature.

\subsection{Experimental parameters}

The gravity currents here simulated are classified as Boussinesq because the density ratio $\gamma=\rho_{\mathrm{c}} / \rho_{\mathrm{a}}$ is close to unity $\left(\rho_{\mathrm{c}}\right.$ is the gravity current initial density in the lock and $\rho_{\mathrm{a}}$ is the density of the ambient water). The experimental parameters of the four currents reproduced are shown in Table 1 where $\mathrm{h}_{0}$ is the initial depth of both salt and fresh water, $\mathrm{g}_{0}$ is the initial reduced gravity of the dense fluid defined as:

$g_{o}=g \frac{\rho_{a}-\rho_{c}}{\rho_{a}}$

Fr is the bulk densimetric Froude number derived for each test as:

$\mathrm{Fr}_{\mathrm{b}}=\frac{\mathrm{u}_{\mathrm{f}}}{\sqrt{\mathrm{g}_{0} \mathrm{~h}_{0}}}$

with $\mathrm{u}_{\mathrm{f}}$ the velocity of propagation of the front and $\mathrm{Re}$ is the Reynolds number determined as:

$\operatorname{Re}_{0}=\frac{\mathrm{u}_{0} \mathrm{~h}_{0}}{\mathrm{v}}$

with $\mathrm{u}_{0}=\sqrt{\mathrm{g}_{0} \mathrm{~h}_{0}}$ the buoyancy velocity.

\subsection{Logarithmic law}

For a smooth boundary, the flow column can be considered as composed by two overlapping regions: the inner and the outer layers (Dey 2014, among many others). It is in the inner region where most of the turbulent energy is produced and transported to the outer region by the so called 'eddy' motions, that in the vicinity of the wall take the form of violent ejection of low-speed fluid (Kline et al. 1967).

Table 1. Experimental parameters for the four tests.

\begin{tabular}{lllllll}
\hline Test & $\begin{array}{l}\mathrm{h}_{0} \\
(\mathrm{~m})\end{array}$ & $\begin{array}{l}\rho_{\mathrm{c}} \\
\left(\mathrm{kg} / \mathrm{m}^{3}\right)\end{array}$ & $\begin{array}{l}\gamma \\
(-)\end{array}$ & $\begin{array}{l}\mathrm{g}_{0} \\
\left(\mathrm{~m}^{2} / \mathrm{s}\right)\end{array}$ & $\begin{array}{l}\mathrm{Fr}_{\mathrm{b}} \\
(-)\end{array}$ & $\begin{array}{l}\mathrm{Re}_{0} \\
(-)\end{array}$ \\
\hline $\mathrm{S} 1$ & 0.20 & 1022 & 0.98 & 0.23 & 0.114 & 42480 \\
$\mathrm{~S} 2$ & 0.20 & 1030 & 0.97 & 0.30 & 0.128 & 48991 \\
$\mathrm{~S} 3$ & 0.20 & 1040 & 0.96 & 0.39 & 0.197 & 55961 \\
$\mathrm{~S} 4$ & 0.20 & 1048 & 0.95 & 0.46 & 0.157 & 60884 \\
\hline
\end{tabular}

Under the assumptions of flow gradually varied in the longitudinal direction, essentially two dimensional in vertical plane, and with high relative submersion, the longitudinal velocity in the overlapping layer can be fitted to the logarithmic law of the wall (Ferreira et al. 2012):

$\frac{\mathrm{u}(\mathrm{z})}{\mathrm{u}_{*}}=\frac{1}{\mathrm{\kappa}} \ln \left(\frac{\mathrm{z}}{\mathrm{z}_{0}}\right)$

where $\mathrm{u}=\mathrm{u}(\mathrm{z})$ is the mean longitudinal velocity (generally averaged over a sufficiently long time scale), $u_{*}$ is the friction velocity, $\kappa$ is the von Kármán constant, $\mathrm{z}$ is the vertical coordinate and $\mathrm{z}_{0}$ is the zero-velocity level.

Here the flow boundary is assumed to be smooth. Thus, the classic value of the von Kármán constant of $\kappa=0.405$ is adopted. Discussions on the estimation of $\kappa$ can be found in Ferreira (2015) where the flow's dependence of this value is analyzed. There is not however a theoretical reasoning to justify the universality of the von Kármán constant, neither its validity to flows with the structure of a density current.

For smooth boundary flows, as herein considered, $z_{0}$ scales with the thickness of the viscous layer (thus with $\mathrm{u}_{*} / v$, where $v$ is the kinematic viscosity of the fluid). For rough-bed flows, it generally corresponds to a characteristic hydraulic roughness length.

The passage of the density current does not correspond to a steady process. However, it is possible to identify time intervals where the flow properties remain (quasi) invariant. We may thus apply a zone-average along the quasi-stationary periods (Franca and Brocchini 2015) to obtain the mean velocity profiles.

\subsection{Fitting procedure}

The equation of the logarithmic law of the wall can be rewritten as:

$\mathrm{u}=\mathrm{A} \ln (\mathrm{z})-\mathrm{B}$

where

$\mathrm{A}=\frac{\mathrm{u}_{*}}{\kappa}, \mathrm{B}=\frac{\mathrm{u}_{*}}{\mathrm{k}} \ln \left(\mathrm{z}_{0}\right)$

Then, by determining the coefficients $\mathrm{A}$ and B through a fitting procedure, one obtains an estimation of $\mathrm{u}_{*}$ which is the velocity scale corresponding to the bed shear stress (Chassaing 2000), determined as $\tau_{b}=\rho u_{*}^{2}$, where $\rho$ is the flow density here considered $\rho_{c}$

The fitting procedure of the logarithmic layer was determined stepwise, extending a linear least square 
fitting range (in a semi-logarithmic scale) from the lowest measured point until the maximum velocity vertical position. The layer providing the best regression coefficient was chosen and considered for the estimation of $\mathrm{u}_{*}$, corresponding to the extent of the logarithmic layer. The fitting procedure is adopted for each instantaneous profile collected with the ADVP instrumentation zone-averaged over a window enough long corresponding to a quasi-steady region. Thus, an estimation of the bed shear stress is made for each measuring instant.

In Figure 3 the fitting procedure for one velocity profile is illustrated corresponding to a regression coefficient of $\mathrm{R}^{2}=0.9944$.

\subsection{Verifications}

The assumptions mentioned earlier for the fitting of the logarithmic law of the wall require to be validated after the estimation of the friction velocity. The assumptions checked are: (i) the smooth-wall assumption; (ii) the validity of a universal von Kármán constant; the (iii) thickness admitted for the overlapping (logarithmic) layer.

The assumption (i) is verified if the shear Reynolds number (or skin roughness, $\mathrm{k}_{\mathrm{s}}$, normalized by the viscous layer) is lesser than 5 :

$\frac{\mathrm{k}_{\mathrm{s}} \mathrm{u}_{*}}{\mathrm{v}} \leq 5$

The Prandtl's mixing layer concept which establishes between $\mathrm{u}_{*}\left(\frac{\mathrm{du}}{\mathrm{d}}\right)^{-1}$ and $\mathrm{z}$ a linear relation in the overlapping layer with slope equal to $\kappa$ (Dey 2014), is used to independently verify the use of a universal von Kármán constant (ii) (see Franca et al. 2008 and Ferreira et al. 2012 for similar procedures applied for rough flows).

The upper limit of the overlapping layer, which is the upper vertical position used for the logarithmic fit $\left(\mathrm{z}_{\mathrm{u}}\right)$, should correspond to a maximum of $\mathrm{z}^{+}=\mathrm{z}_{\mathrm{u}}^{+}=\frac{\mathrm{z}_{\mathrm{u}} \mathrm{u}_{*}}{v}=500$ (Chassaing 2000).

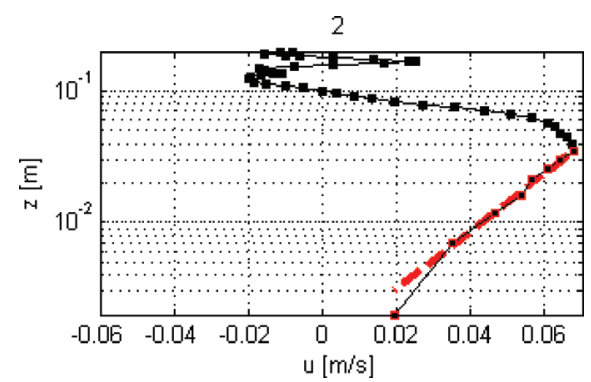

Figure 3. Logarithmic profile method for one of the velocity profile. The red dashed line linearly fits the velocity measurements within the determined logarithmic layer.

\section{RESULTS}

\subsection{Flow velocities}

The velocity data collected consists of instantaneous $3 \mathrm{D}$ velocity profiles along a vertical. To allow for a spatial visualization of the different regions composing the current, i.e. head, body and tail, and to relate these with the bed shear stress, the information in time is converted into space. This is done by considering a constant advection velocity equal to the front velocity.

The instantaneous longitudinal velocity fields for the four tests performed are showed in Figure 4. Increasing initial gravity current density results in larger longitudinal velocities. This brings advantages in the signal to noise ratio and it is visible by the quality of the signals in Figure 4 which is increasing with the density. For low flow velocities the base noise inherent to the Doppler instrumentation superimposes to the Doppler frequency corresponding to the measured flow velocity.

While the current advances, a return flow forms on the upper ambient fluid layer. This countercurrent shows a jet-flow configuration that becomes more defined for higher gravity current velocities. There is a time scale for the complete formation of the return flow which is dependent on the initial density in the lock, and on the inertia of the physical system composed by the experimental channel and the large downstream reservoir.

The gravity current regions are recognizable in Figure 4: the unsteady and highly turbulent front (or head) followed by the body of the current.

The procedure above explained is thus applied to the measurements in order to obtain mean velocity profiles for each instant, and then the corresponding friction velocity. Each mean velocity profile is the result of a zone-averaged over 1 second.

Figure 5 shows all the mean velocities as a function of the flow depth within the overlapping layer for the profiles of test S3. The data collapse linearly when plotting the distance from the bed in the logarithmic scale and it indicates the consistency of the application of the method to the measurements (Sequeiros et al. 2010).

\subsection{Bed shear stress}

After the estimation of the friction velocity by means of the logarithmic profile method, the bed shear stress is calculated. The evolution of the bed shear stress along the streamwise direction is shown in Figure 6 over the current velocity as a background. The red line indicates the bed shear stress calculated for each instantaneous profile.

There is not a clear tendency between the amplitude of the bed shear stress values and the density. In the regions of higher longitudinal gradient of 

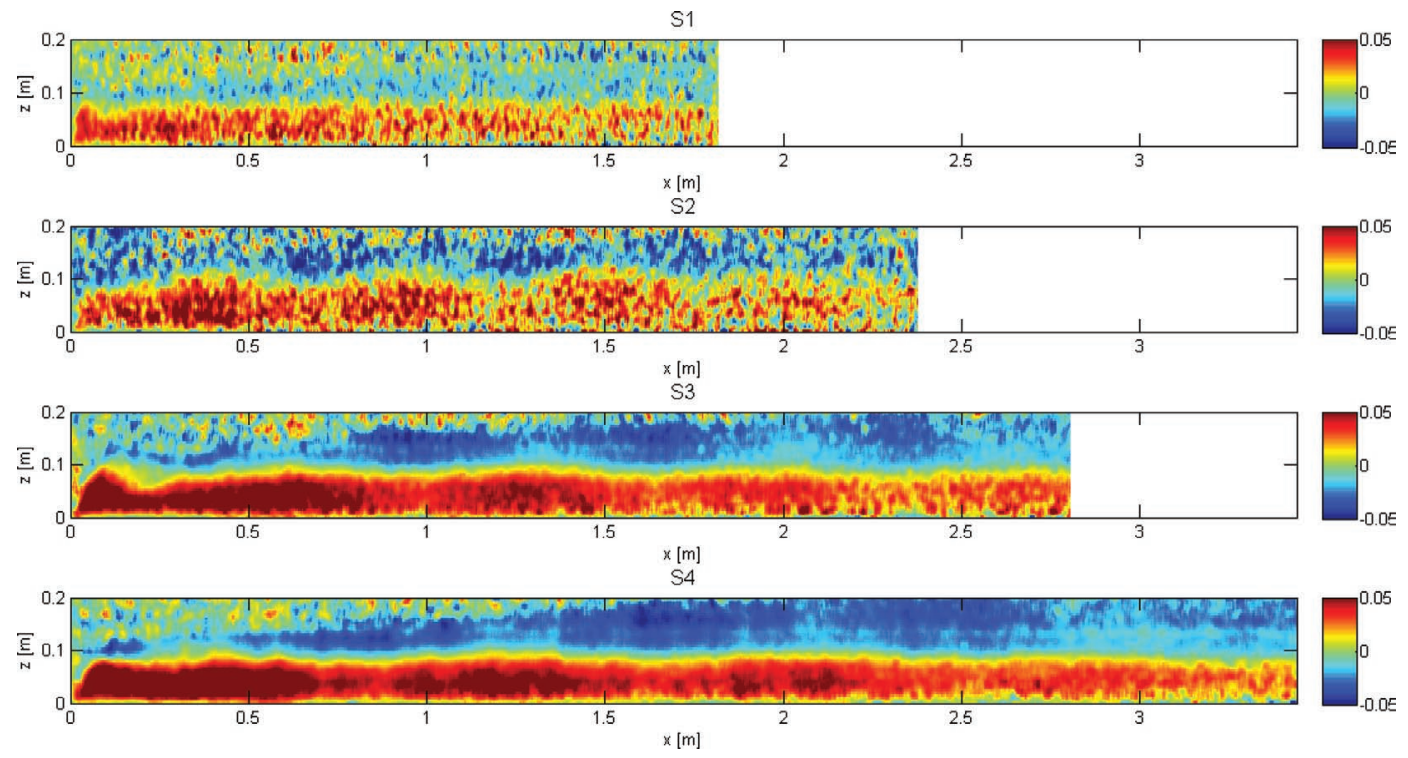

Figure 4. Instantaneous longitudinal velocity field $(\mathrm{m} / \mathrm{s})$ for the four tests of increasing initial density.

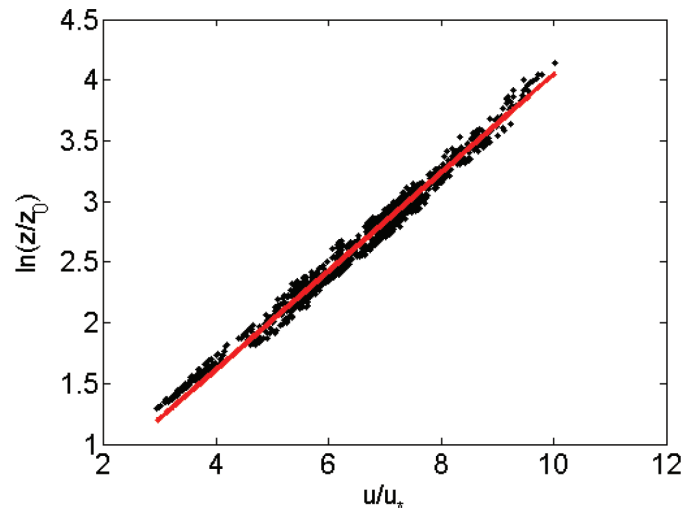

Figure 5. Collapsed near-bed profiles for test S3 showing the consistency of linear interpolation.

the velocity we may observed the largest peaks in the bed shear stress. The head of the current typically exhibits high values of bed shear stress. After the passage of the head, two peaks are visible for three out of the four experiments.

\subsection{Verifications}

The verification of assumption (i) (section 2.6) was made by considering a skin roughness of the channel bottom of $\mathrm{k}_{\mathrm{s}}=0.2 \mathrm{~mm}$, which corresponds to PVC. It was verified that the relation between this and the viscous layer scale was always below the limit of 5 .
A backward estimation of the von Kármán constant $\kappa$ have been done to evaluate the validity of assumption (ii) (section 2.6) as enumerated before. For a finer assessment of the friction velocity this is in fact of crucial importance. From the definition of mixing length (1) given by Prandtl and assuming that this is proportional to the distance from the bed ( $\mathrm{z}$ ) through the von Kármán constant $\kappa($ Dey 2014):

$1=\kappa Z$

The total bed shear stress can be written as:

$\tau_{\mathrm{b}}=\rho u_{*}\left(\frac{\mathrm{du}}{\mathrm{dz}}\right)$

With the definition of friction velocity $\left(\tau_{\mathrm{b}}=\rho \mathrm{u}_{*}^{2}\right)$, the bed shear stress can be calculated:

$1=u_{*}\left(\frac{d u}{d z}\right)^{-1}$

Equation (8) and (10) are finally combined giving the following relation:

$u_{*}\left(\frac{d u}{d z}\right)^{-1}=\kappa z$

This allows for an independent evaluation of the von Kármán constant. In Figure 7 this procedure 

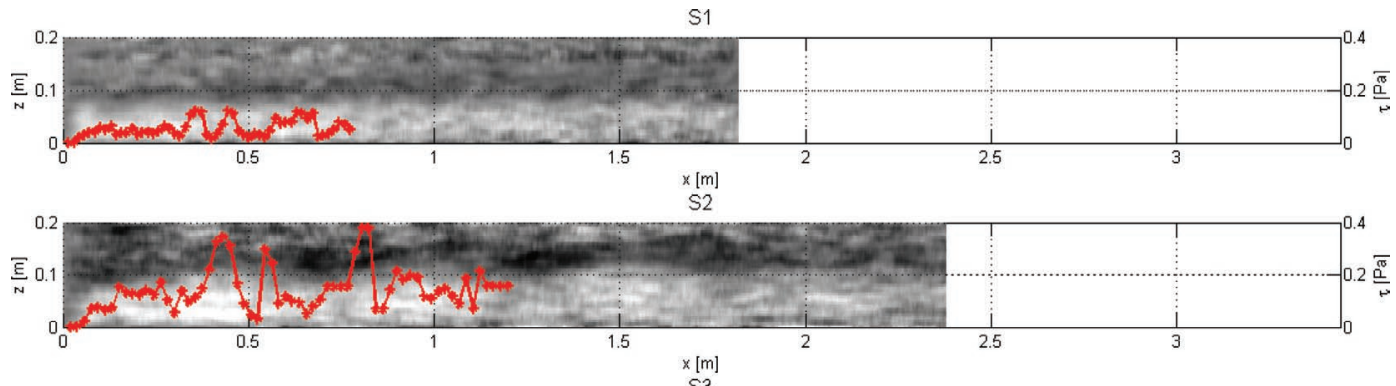

53
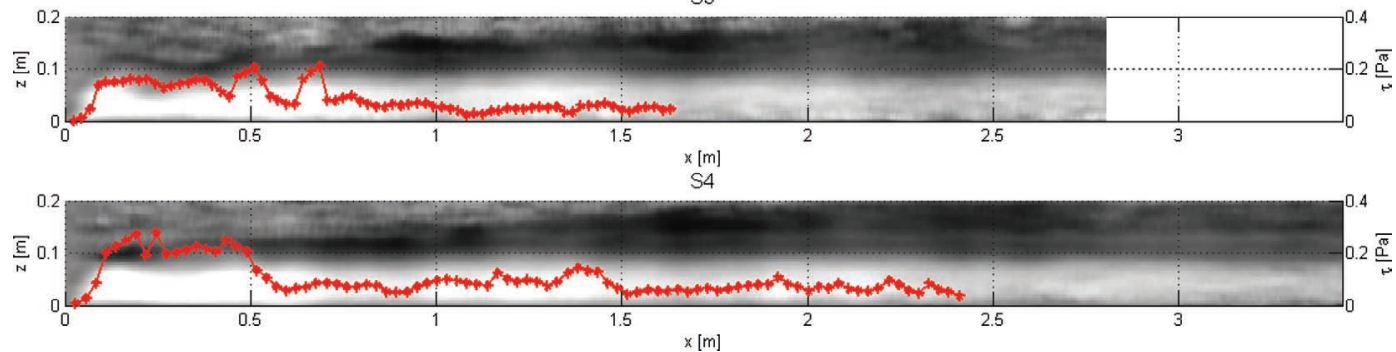

Figure 6. Bed shear stress evolution computed after the fitting procedure of the log layer.
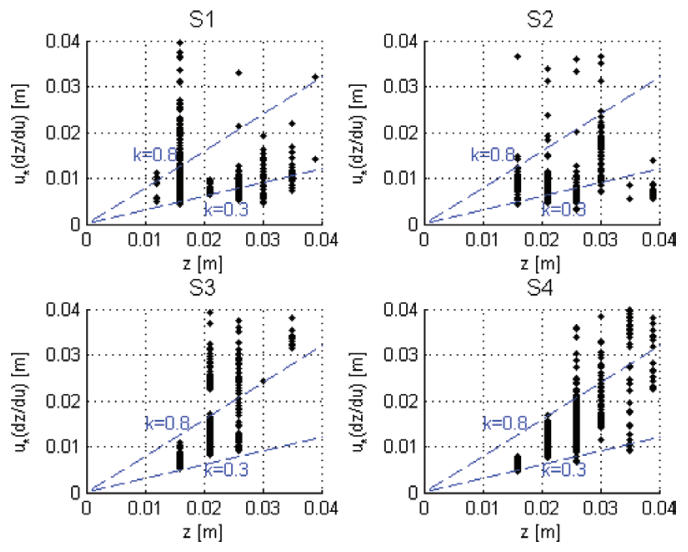

Figure 7. Determination of the von Kármán constant using Equation (11).

is applied considering the velocity profiles included in the body of the currents. The velocity gradient was calculated from a two point running mean in the vertical (Franca et al. 2008). The two dashed lines delimited a zone where the constant has values comprised between 0.3 and 0.8 . Despite the dispersion, a tendency is clear and the values spread around the value of 0.405 derived by von Kármán.

Assumption (iii) (section 2.6) was also checked and we verified that for the estimated values of the friction velocity, $\mathrm{z}_{\mathrm{u}}^{+}$was always below 500 .

\section{CONCLUSIONS}

In this study, the estimation of bed shear stress of gravity currents over smooth bed was made and discussed. The gravity currents corresponded to large volume of release and were generated by lock-exchange experiments. The high-resolution 3D-ADVP provides velocity data that, with the application of the logarithmic profile method, can be used to estimate the friction velocity from which the bed shear stress can be derived. The spatial distribution of the bed shear stress in saline density currents was estimated for four tests with different initial excess density.

The mean velocity profiles used to estimated bed shear stress were obtained by zone-averaging the instantaneous over a constant time interval of 1 second. The use of a constant time window is maybe limiting and should be revisited. When the flow is highly unsteady a shorter time window could be procured, although with the risk of being not correct due to the incapacity of producing adequate flow statistics.

In steadier regions of the current, the use of a too small window may be jeopardizing the visualization of the evolution of the bed shear stress. This can be the case of the fourth test where no peaks are visible in the bed shear stress distribution after the passage of the current head.

The preliminary results herein shown can further be explored and applied to evaluate the capacity of entrainment and detrainment on density currents when passing over mobile beds. 


\section{ACKNOWLEDGMENTS}

This research was funded by the European project SEDITRANS funded by Marie Curie Actions, FP7-PEOPLE-2013-ITN-607394 (Multi partnerInitial Training Networks).

\section{REFERENCES}

Adduce, C., Sciortino, G. \& Proietti, S. 2012. Gravity Currents Produced by Lock Exchanges: Experiments and Simulations with a Two-Layer Shallow-Water Model with Entrainment. Journal of Hydraulic Engineering, 138(2): 111-121.

Altinakar, S., Graf, W.H. \& Hopfinger, E.J. 1990. Weakly depositing turbidity current on a small slope. Journal of Hydraulic Research, 28(1): 55-80.

Bagherimiyab, F. \& Lemmin, U. 2013. Shear velocity estimates in rough-bed open-channel flow. Earth Surface Processes and Landforms, 38(14): 1714-1724.

Blanckaert, K. \& Lemmin, U. 2006. Means of noise reduction in acoustic turbulence measurements. Journal of Hydraulic Research, 44(1): 3-17.

Britter, R.E. \& Linden, P.F. 2006. The motion of the front of a gravity current travelling down an incline. Journal of Fluid Mechanics, 99(03): 531.

Chassaing P. 2000. Turbulence en Mécanique des Fluides. Cépaduès-Éditions, Toulouse.

Cossu, R. \& Wells, M.G. 2012. A comparison of the shear stress distribution in the bottom boundary layer of experimental density and turbidity currents. European Journal of Mechanics, B/Fluids, 32(1): 70-79.

Dey S. 2014. Fluvial hydrodynamics: hydrodynamic and sediment transport phenomena. Springer, Berlin.

Ferreira, R.M.L., Franca, M.J., Leal, J.G. a B. \& Cardoso, A.H. 2012. Flow over rough mobile beds: Friction factor and vertical distribution of the longitudinal mean velocity. Water Resources Research, 48(5): $1-14$.

Ferreira, R.M.L. 2015. The von Kármán constant for flows over rough mobile beds. Lessons learned from dimensional analysis and similarity. Advances in Water Resources, 81: 19-32.

Franca, M.J., Brocchini, M. 2015. Turbulence in Rivers. Rivers-Physical, Fluvial and Environmental Processes, Springer International Publishing: 51-78.

Franca, M.J., \& Lemmin, U. 2006. Eliminating velocity aliasing in acoustic Doppler velocity profiler data. Measurement Science and Technology, 17(2): 313-322.

Franca, M.J., Ferreira, R.M.L., \& Lemmin, U. 2008. Parameterization of the logarithmic layer of doubleaveraged streamwise velocity profiles in gravel-bed river flows. Advances in Water Resources, 31(6): 915-925.

Garcia, M. \& Parker, G. 1993. Experiments on the entrainment of sediment into suspension by a dense bottom current. Journal of Geophysical Research, 98: 4793-4807.

Goring, D.G., \& Nikora, V.I. 2002. Despiking Acoustic Doppler Velocimeter Data. Journal of Hydraulic Engineering, 128(1): 117-126.
Gray, T.E., Alexander, J., \& Leeder, M.R. 2006. Longitudinal flow evolution and turbulence structure of dynamically similar, sustained, saline density and turbidity currents. Journal of Geophysical Research: Oceans, 111(8): 1-14.

Huppert, H.E., \& Simpson, J.E. 1980. The slumping of gravity currents. Journal of Fluid Mechanics, 99(04): 785-799.

Kline, S.J., Reynolds, W.C., Schraub, F.a., \& Rundstatler, P.W. 1967. The structure of turbulent boundary layers. Journal of Fluid Mechanics, 30: 741-773.

Lemmin, U., \& Rolland, T. 1997. Acoustic velocity profiler for laboratory and field studies. Journal of Hydraulic Engineering, (12): 1089-1098.

Lombardi, V., Adduce, C., Sciortino, G. \& La Rocca, M. 2015. Gravity currents flowing upslope: Laboratory experiments and shallow-water simulations. Phys. Fluids, 27, 016602.

Nogueira, H.I.S., Adduce, C., Alves, E., \& Franca, M.J. 2013. Analysis of lock-exchange gravity currents over smooth and rough beds. Journal of Hydraulic Research, 51(4): 417-431.

Nogueira, H.I.S., Adduce, C., Alves, E., \& Franca, M.J. 2014. Dynamics of the head of gravity currents. Environmental Fluid Mechanics, 14(2): 519-540.

Ooi, S.K., Constantinescu, G., \& Weber, L. 2009. Numerical simulations of lock-exchange compositional gravity current. Journal of Fluid Mechanics, 635: 361-388.

Ottolenghi, L., Adduce, C., Inghilesi, R., Roman, F. \& Armenio, V. 2014. Large Eddy Simulation of gravity currents moving on up-sloping boundaries. River flow, 2014, 3-5 September 2014, Lausanne.

Parker, G., Fukushima, Y. \& Pantin, H.M. 1986. Selfacceler ating turbidity currents. Journal of Fluid Mechanics, 171: 145-181.

Rottman, J.W., \& Simpson, J.E. 1983. Gravity currents produced by instantaneous releases of a heavy fluid in a rectangular channel. Journal of Fluid Mechanics, 135: 95-110.

Sequeiros, O.E., Spinewine, B., Beaubouef, R.T., Sun, T., Garcia, M.H., \& Parker, G. 2010. Bedload transport and bed resistance associated with density and turbidity currents. Sedimentology, 57(6): 1463-1490.

Shin, J.O., Dalziel, S.B. \& Linden, P.F. 2004. Gravity currents produced by lock exchange. Journal of Fluid Mechanics, 521: 1-34.

Simpson, J.E. 1997. Gravity Currents in the Environment and the Laboratory. Cambridge University Press.

Tokyay, T., Constantinescu, G., \& Meiburg, E. 2011. Lock-exchange gravity currents with a high volume of release propagating over a periodic array of obstacles. Journal of Fluid Mechanics, 672: 570-605.

Tokyay, T., Constantinescu, G., \& Meiburg, E. 2012. Tail structure and bed friction velocity distribution of gravity currents propagating over an array of obstacles. Journal of Fluid Mechanics, 694: 252-291.

Yang, S., Dharmasiri, N. Chamini. \& Han, Y. 2012. Momentum balance method and estimation of boundary shear stress distribution. Journal of Hydraulic Engineering, 138(7): 657-660. 